\title{
Increasing the Conductivity and Adhesion of Polypyrrole Hydrogels with Electropolymerized Polydopamine
}

\author{
Supporting Information
}

Evelyn Chalmers, ${ }^{\dagger}$ Haeshin Lee,${ }^{\ddagger}$ Chuang Zhu, ${ }^{\dagger}$ and Xuqing Liu ${ }^{\dagger *}$

†+Department of Materials, University of Manchester, Oxford Road, Manchester M13 9PL

¥Department of Chemistry, Korea Advanced Institute of Science and Technology, Daejeon 34141 , Republic of Korea

E-mail: xuqing.liu@manchester.ac.uk 
Figure A: SEM images of: a) A $10 \mathrm{mV} / \mathrm{s}$, b) B $100 \mathrm{mV} / \mathrm{s}$, c) Ppy gel after charge cycling in $\mathrm{NaCl}$ electrolyte, d) Ppy-dopamine-tris gel after cycling in $\mathrm{NaCl}$ electrolyte to produce a polydopamine phase.

(a)

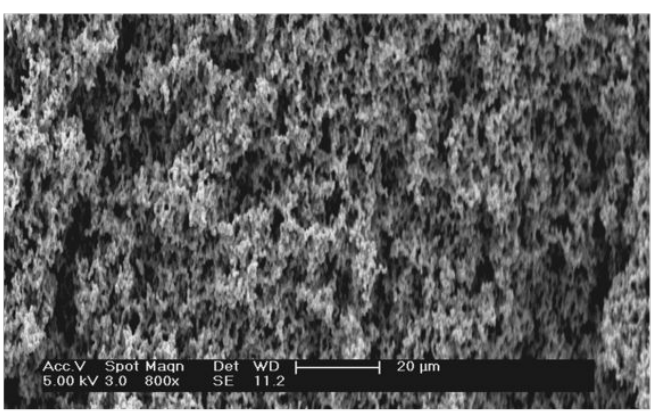

(c)

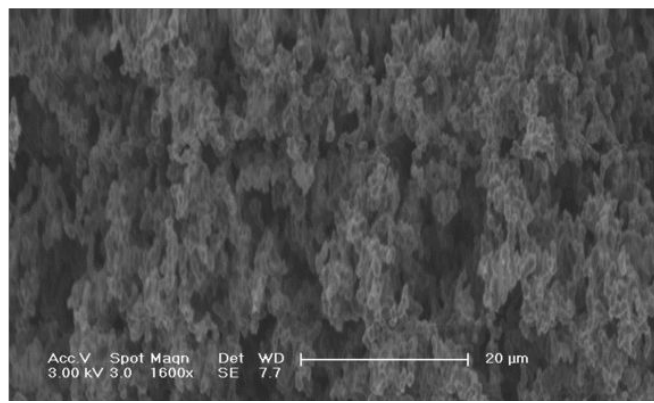

(b)

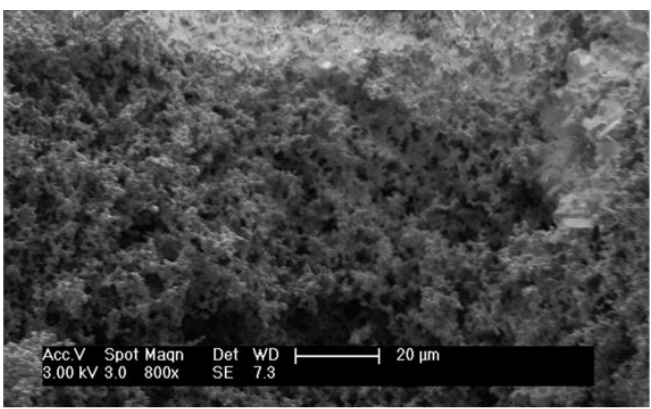

(d)

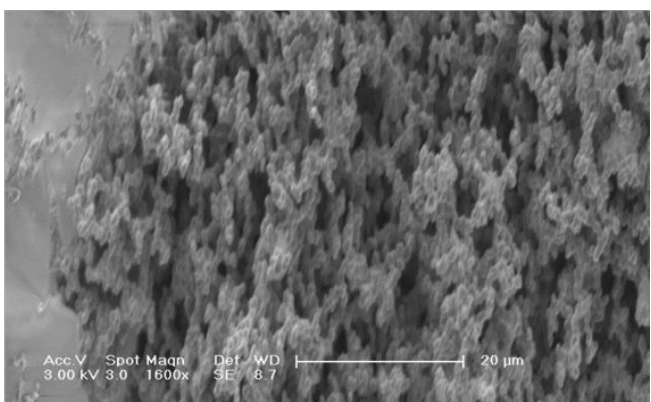

Figure B: SEM images of: a) polypyrrole gel before charge cycling, b) the same gel after charge cycling, note formation of non-conducting $\mathrm{NaCl}$ surface layer, c) polypyrrole-polydopamine gel.

(a)

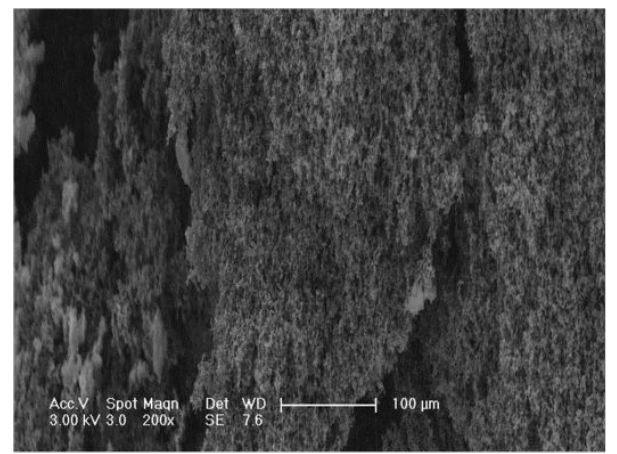

(b)

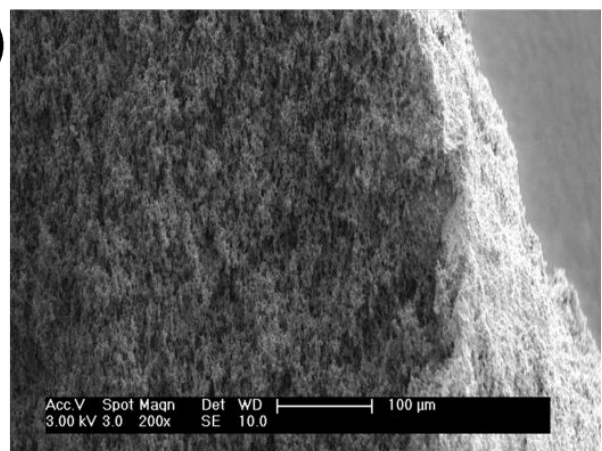

(c)

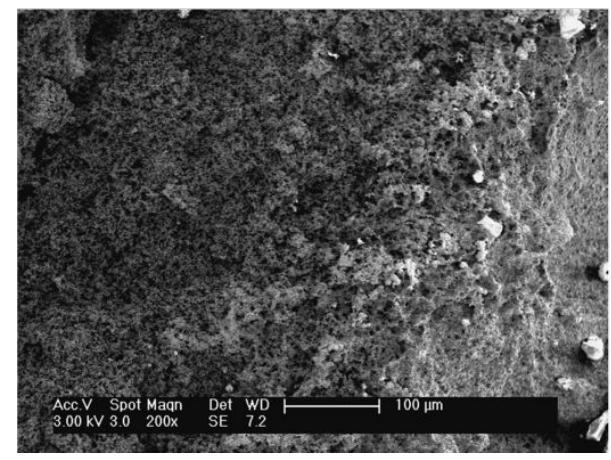


Figure C: Raman spectra of a) polypyrrole charge cycled with dopamine and tris in electrolyte; with proportions as discussed in the manuscript, b) with dopamine and/or tris added to pyrrole/ $\mathrm{Fe}\left(\mathrm{NO}_{3}\right)_{3}$.
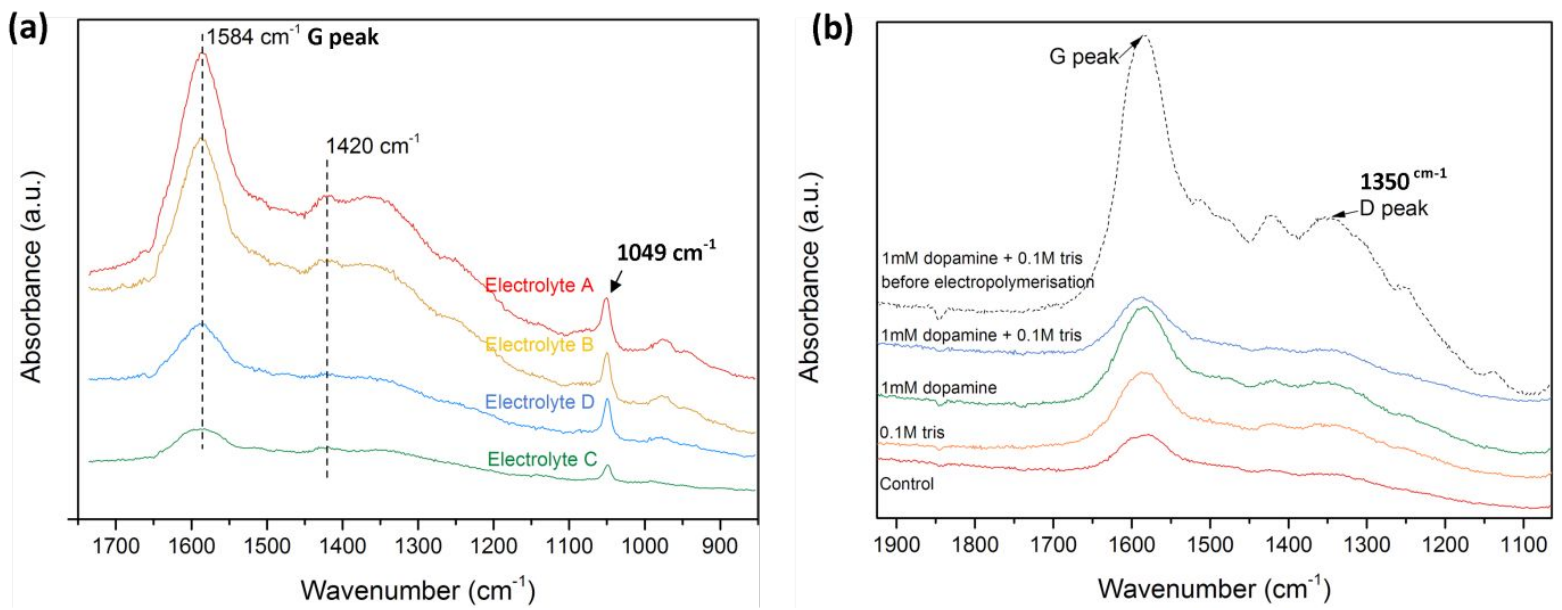

The broad peak with a centre at $1350 \mathrm{~cm}^{-1}$ corresponds to sp3 C-C bonding. This peak increased in height when a faster scan rate was used for electropolymerisation, indicating that a lower dopamine concentration and slower scan rate lead to less disruption of both dopamine and polypyrrole aromatic structures. The distinct peak at $1049 \mathrm{~cm}^{-1}$ corresponds to the bipolaron state of polypyrrole, indicating that the conductivity of polypyrrole is unaffected by the presence of tris in the electrolyte. The Raman spectra of the hydrogels containing dopamine and/or tris within the gel also indicate a change in the proportion of the dopamine derivatives from charge cycling. The peaks seen only on the pre-electropolymerised gel containing both tris and dopamine occur at $1518 \mathrm{~cm}^{-1}$ from an [NO2]- stretch from the polypyrrole dopant; and $1482 \mathrm{~cm}^{-1}$ from the aromatic ring of the dopamine monomer. The latter peak is also seen in the dopamine-pyrrole spectrum; its loss after charge cycling may indicate oxidation of the dopamine aromatic ring to dopaminechrome or 5,6indolequinone. Most significantly, peaks corresponding to a $\mathrm{CH} 2 / \mathrm{CH} 3$ symmetric stretch $\left(1421 \mathrm{~cm}^{-1}\right)$ and a trisubstituted amine $\left(1251 \mathrm{~cm}^{-1}\right)$ within this spectrum are also lost after electropolymerisation, indicating that cyclization of the dopamine to leukodopaminechrome is induced through charge cycling. Between pre-and post-electropolymerisation spectra, the ratio of the G/D peak areas remains constant, indicating preservation of polypyrrole bond conjugation. 
Figure D: Charge cycling of polypyrrole gels with dopamine and tris in the $4.0 \mathrm{M} \mathrm{NaCl} . \mathrm{KCl}$ electrolyte. Cycling was performed five times, at $10 \mathrm{mV} / \mathrm{s}$ scan rate. a) with electrolyte $\mathrm{A}(0.1 \mathrm{M}$ tris, $1 \mathrm{mM}$ dopamine), b) electrolyte $B, c$ ) electrolyte $C, d$ ) electrolyte $D$. The evolution of relevant peaks is marked.
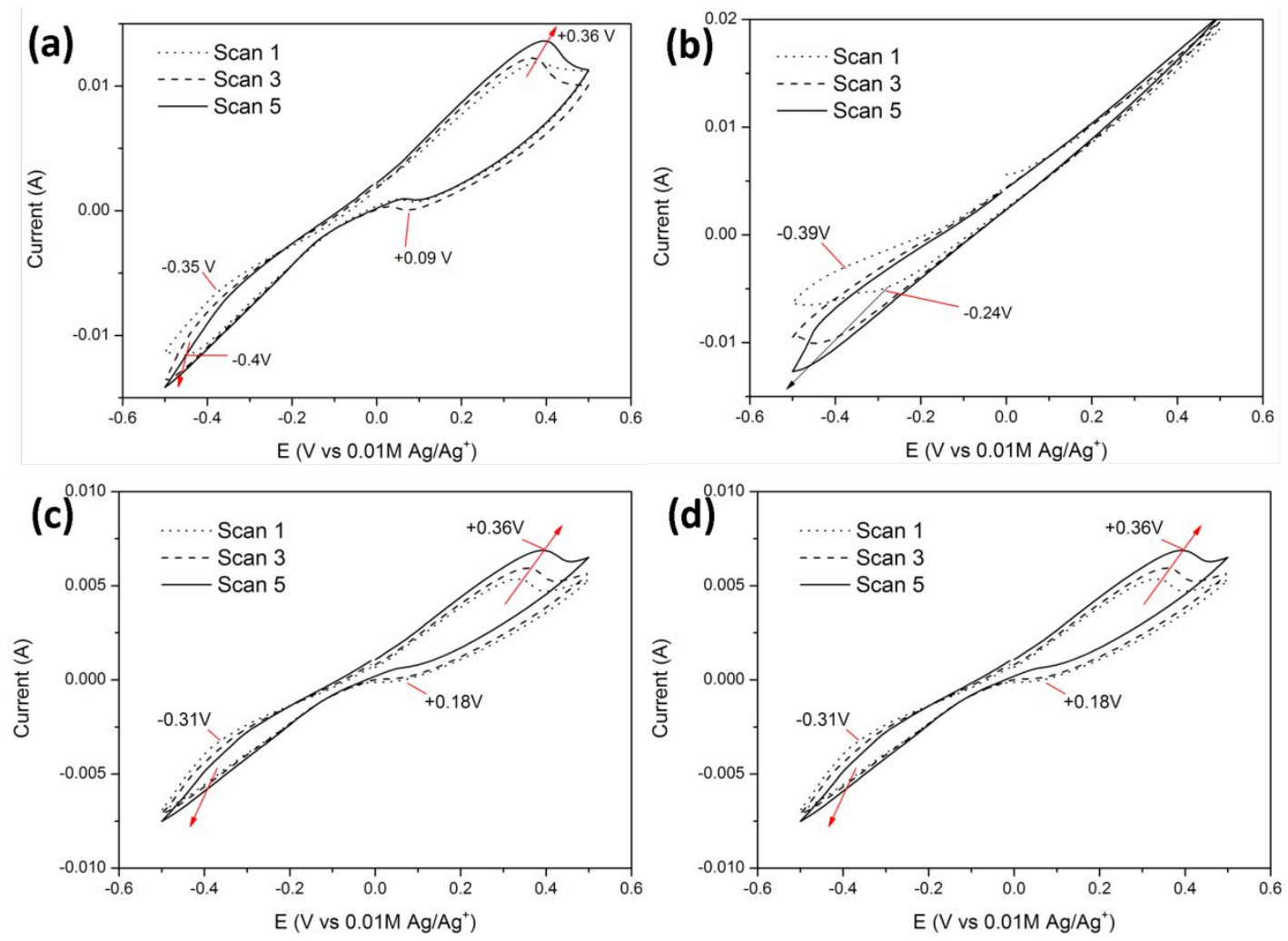

All gels shown in these plots were electropolymerised at a scan rate of $10 \mathrm{mV} \mathrm{s}^{-1}$. With electrolytes $\mathrm{A}, \mathrm{C}$, and $\mathrm{D}$, a large peak can be seen to evolve at $+0.36 \mathrm{~V}$, which grows in size and shifts to $+0.4 \mathrm{~V}$ with an increasing scan number. This corresponds to the oxidation of dopamine to dopaminequinone.[1] This peak may not appear in scan B due to a higher concentration of unoxidised dopamine in solution acting to obscure any oxidation peak. Salomäki's research notes an oxidation peak at $+0.32 \mathrm{~V} \mathrm{Ag} / \mathrm{AgCl}$ at pH 4.5 , corresponding to the two-electron oxidation potential of the dihydroxy/leukodopaminechrome molecule to the quinone form: this peak may be shifted in our results due to the presence of $\mathrm{Fe}^{3+}$ ions, and a lower $\mathrm{pH}$ of 2.5 arising from excess $\mathrm{H}^{+}$ions produced during polypyrrole formation.[2]

The peaks seen in Figure $\mathrm{D}$ occurring between $+0.09 \mathrm{~V}$ and $+0.18 \mathrm{~V}$ are therefore likely to correspond to the one-electron oxidation potential of the same equilibrium. The reduction peak seen at $-0.4 \mathrm{~V}$ is the reduction of dopaminequinone back to dopamine - this peak is also observed when dopamine is incorporated without tris into the polypyrrole hydrogel prior to electropolymerisation, but is not observed when dopamine and tris are incorporated into the pyrrole prior to gel formation (see Figure 3 a) in the main manuscript.

However, an absence of all three peaks occurred when a higher ratio of tris:dopamine was used in the electrolyte. This may be due to tris directing oxidation of the dopamine molecules through formation of hydrogen bonds with dopamine's hydroxyl and amine groups, which will alter the oxidation mechanism of dopamine to dopaminequinone and leukodopaminechrome. The peak 
corresponding to the reduction of $\left[\mathrm{NO}_{3}\right]^{-}$doped polypyrrole to the non-conducting form is seen in wider scans at $-0.57 \mathrm{~V}$. All CV curves show an increase in conductivity with increasing number of charge cycles due to incorporation of $\mathrm{Cl}^{-}$anions from the electrolyte into the hydrogel with each successive voltage sweep, wherein they provide additional doping to the polypyrrole.

Figure E: Charge cycling of polypyrrole gels in $4.0 \mathrm{M} \mathrm{NaCl} . \mathrm{KCl}$ solution, again for five scans at $10 \mathrm{mV} / \mathrm{s}$ scan rate; a) pure polypyrrole gel, b) polypyrrole $+0.1 \mathrm{M}$ tris added before pyrrole polymerisation, c) polypyrrole $+1 \mathrm{mM}$ dopamine added before pyrrole polymerisation .
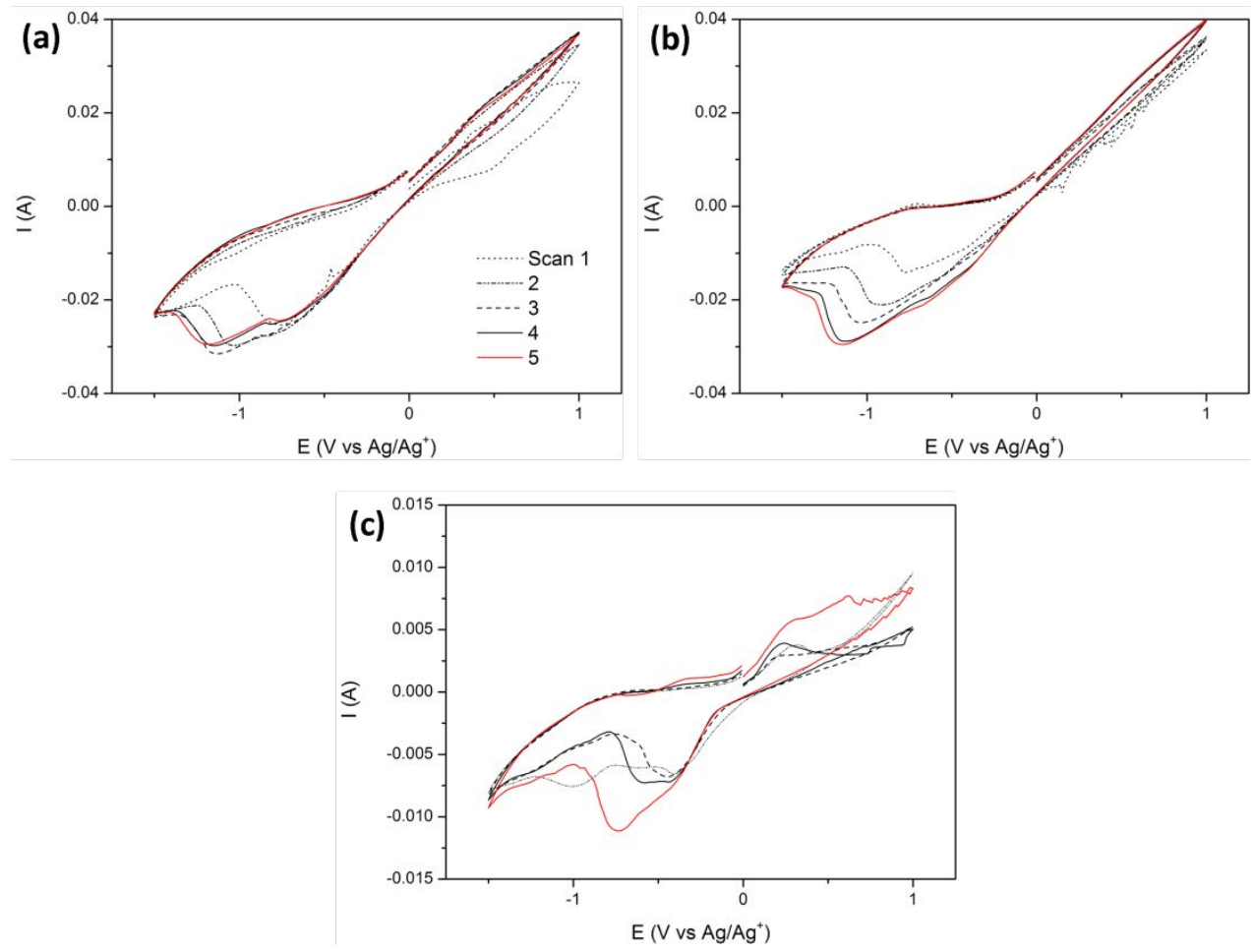

The addition of $1 \mathrm{mM}$ of dopamine to the pyrrole prior to chemical polymerisation produced a lower anodic current throughout, likely due to disruption and misalignment of the polypyrrole chain by $\pi$ bonding with dopamine, which impeded bipolaron movement. This is also seen as a decrease in the gels' conductivity prior to charge cycling (Figure $3 \mathrm{~b}$ ). [3] An oxidation peak now occurred at $+0.29 \mathrm{~V}$ corresponding to oxidation of dopamine to dopaminequinone.[4] Wang's research on dopamine electropolymerisation[5] exhibited similar CV curves; the peak here occurred $0.2 \mathrm{~V}$ higher, likely due to improved stabilisation of dopamine by polypyrrole via $\pi-\pi$ interactions. The peak occurring at $+0.39 \mathrm{~V}$ is the oxidation of dopamine to dopaminequinone, whilst the oxidation peak at $+0.47 \mathrm{~V}$ is the oxidation of leukodopaminechrome to dopaminechrome.[6] An absence of the corresponding reduction peaks indicates that these equilibria are being pushed in the direction of oxidation, cyclization, and polymerisation by the applied potential. The positive shift in the reduction peak of polypyrrole (from $-0.97 \mathrm{~V} \mathrm{Ag} / \mathrm{AgCl}$ to $-0.71 \mathrm{~V} \mathrm{Ag} / \mathrm{AgCl}$ ) is likely due to convolution with the peak corresponding to the reduction of dopaminechrome back to leukodopaminechrome (which occurs at $-0.58 \mathrm{~V}$ ), as well as some replacement of the nitrate dopant with dopamine - making it easier for nitrate to be removed from the gel.[7-9] 
Unlike Wang's and Li's work, we saw an increase in peak current with increasing scan number for all additions to the hydrogel. As can be seen in Figure \ref\{fig:Fig3\} a) in the main text and Figure E in the Supporting Information, this is an indication that the polydopamine formed is electrically conducting. This may be due to covalent bonding of the 5,6-dihydroxyindole (and similar) molecules, leading to $\mathrm{C}-\mathrm{C}$ bond conjugation along a chain of polydopamine rather than $\pi$-stacking.[10]

Figure F: DMA compression of polypyrrole gels (before charge cycling) showing behaviour analogous to an open-pore elastic-plastic foam.

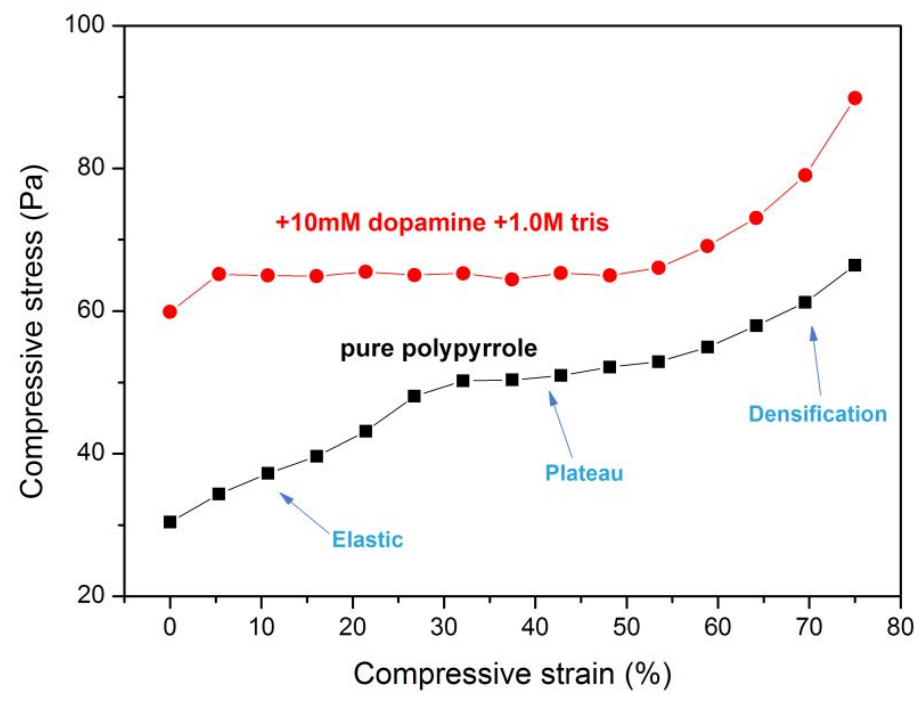

Figure G: tensile test of pure polypyrrole hydrogels (not charge cycled) with moisture added every $2.5 \%$ extension, to minimise drying out and fracture of the samples at low strain.

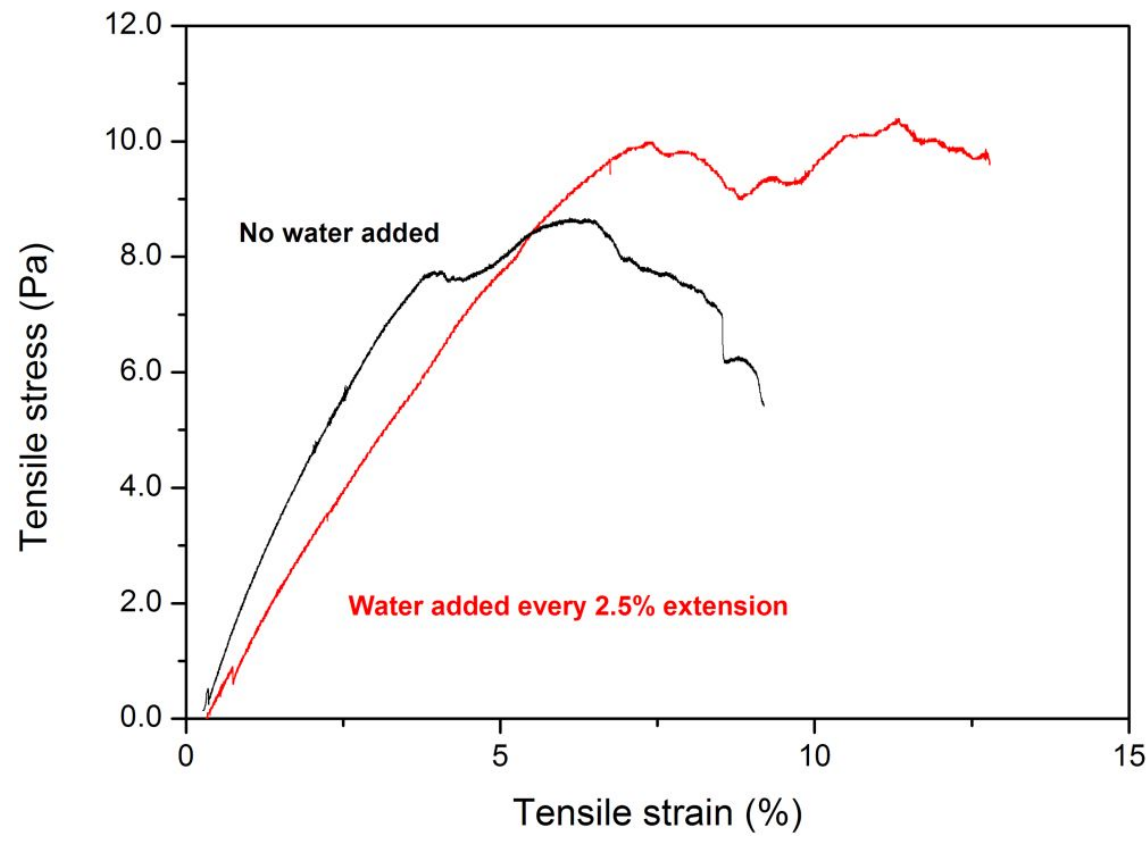


Figure H: FTIR spectra showing the change after charge-cycling and growing polydopamine, with a shift indicating a shift from $3180 \mathrm{~cm}^{-1}$ to $3340 \mathrm{~cm}^{-1}$, indicating a change of the $-\mathrm{OH}$ group of dopamine to the $-\mathrm{OH}$ group of 5,6-dihydroxyindole.

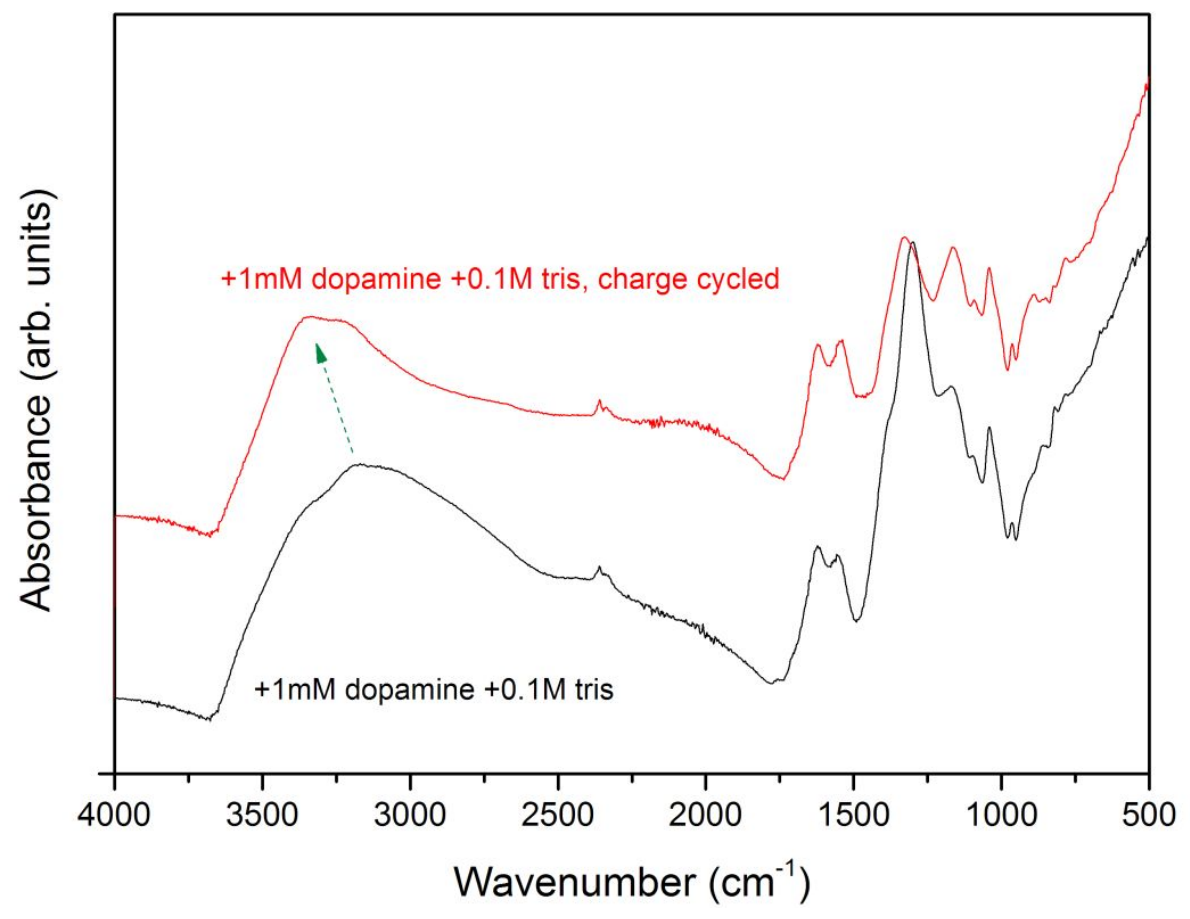

1. Wei, Q., Zhang, F., Li, J., Li, B., Zhao, C., Oxidant-induced dopamine polymerization for multifunctional coating, Polymer Chemistry, 2010, 1, p.1430-1433

2. Shi, Y., Peng, L., Ding, Y., Zhao, Y., Yu, G. Nanostructured conductive polymers for advanced energy storage, Chem. Soc. Rev., 2015, 44, p.6684-6696

3. Almeida, L.C., Correia, R.D., Marta, A., Squillaci, G., Morana, A., La Cara, F., Correia, J.P., Viana, A.S. Electrosynthesis of polydopamine films - tailored matrices for laccase-based biosensors, Applied Surface Science, 2019, 480, p.979-989,

4. Li, S., Wang, H., Young, M., Xu, F., Cheng, G., Cong, H. Properties of Electropolymerized Dopamine and Its Analogues, Langmuir, 2019, 5(35), p.1119-1125

5. Wang, J., Li, B., Li, Z., Ren, K., Jin, L., Zhang, S., Chang, H., Sun, Y., Ji, J., Electropolymerization of dopamine for surface modification of complex-shaped cardiovascular stents, Biomaterials, 2014, 35(27), p.7679-7689

6. Li, Y., Liu, M., Xiang, C., Xie, Q., Yao, S., Electrochemical quartz crystal microbalance study on growth and property of the polymer deposit at gold electrodes during oxidation of dopamine in aqueous solutions, Thin Solid Films, 2006, 497(1), p.270-278

7. Wang, Z., Zeng, J., Tan, G., Liao, J., Zhou, L., Chen, J., Yu, P., Wang, Q., Ning, C. Incorporating catechol into electroactive polypyrrole nanowires on titanium to promote hydroxyapatite formation, Bioactive Materials, 2018, $3(1)$, p.74-79

8. Kim, S., Jang, L., K. Park, H.S., Lee, J.Y. Electrochemical deposition of conductive and adhesive polypyrroledopamine films, Scientific Reports, 2016, 6, p.1-8

9. Zhang, W., Yang, F.K., Pan, Z., Zhang, J., Zhao, B. Bio-inspired dopamine functionalization of polypyrrole for improved adhesion and conductivity, Macromolecular Rapid Communications, 2014, 35(3), p.350-354

10. Ryu, J.H., Messersmith, P.B., and Lee, H. Polydopamine Surface Chemistry: A Decade of Discovery, ACS Applied Materials and Interfaces, 2018, 10(9), p.7523-7540 